\title{
Non-negative spectrum of a digraph
}

\author{
Irena M. Jovanović * \\ School of Computing, Union University, Knez Mihailova 6, 11000 Belgrade, Serbia
}

Received 8 June 2014, accepted 29 March 2016, published online 26 November 2016

\begin{abstract}
Digraphs are considered by means of eigenvalues of the matrix $A A^{T}$, and similarly $A^{T} A$, where $A$ is the adjacency matrix of a digraph. The common spectrum of these matrices is called non-negative spectrum or $N$-spectrum of a digraph. Several properties of the $N$-spectrum are proved. The notion of cospectrality is generalized, and some examples of cospectral (multi)(di)graphs are constructed.
\end{abstract}

Keywords: Digraph, non-negative spectrum, multigraph, cospectrality, isomorphism.

Math. Subj. Class.: 05C20

\section{Introduction}

Spectral (di)graph theory means usage of linear algebra tools and techniques in the study of (di)graphs. It is a very well developed mathematical field (see [8] or [6]) with many applications (see, for example, [2] and [15]).

For any (di)graph matrix $M$, one can build a spectral (di)graph subtheory, and then be able to study (di)graphs by means of eigenvalues of the matrix $M$. We will denote these eigenvalues $M$-eigenvalues. In general case, in order to avoid confusion, to any notion in the corresponding subtheory a prefix ' $M$ ' should be added. Frequently used graph matrices are the adjacency matrix $A$, the Laplacian $L=D-A$ and the signless Laplacian $Q=D+A$, where $D$ is a diagonal matrix of vertex degrees. The spectral (di)graph theory then consolidates all these particular subtheories together with interaction tools.

In this paper, digraphs are considered by means of eigenvalues of the matrix $A A^{T}$, and similarly $A^{T} A$, where $A$ is the adjacency matrix of a digraph. The common spectrum of these matrices is denoted $N$-spectrum and called non-negative spectrum of a digraph. According to [5], the $N$-spectrum of a digraph was not considered in the mathematical

\footnotetext{
${ }^{*}$ The work is supported by Serbian Ministry of Education, Science and Technological Development, Projects 174033 and III45003.

E-mail address: irenaire@gmail.com (Irena M. Jovanović)
} 
literature so far. Since the matrices $A A^{T}$ and $A^{T} A$ appear in applications (see, for example, [11] and [12]), we believe that introduced notion and presented results could be useful to mathematicians and informaticians. Namely, $N$-spectrum can facilitate the examination of digraphs since frequently used adjacency matrix of a digraph is not symmetric in general case, and therefore its spectrum consists of complex numbers. It is well known that digraphs serve as models for different processes and phenomena in computer sciences, where some spectrally based techniques are used in investigations. By this approach some new conclusions and comparisons of existing results could be made.

The paper is organized as follows:

In Section 2 basic digraph terminology is given and some elementary facts related to the matrices $A A^{T}$ and $A^{T} A$ and their spectrum are pointed out. Since this paper represents the first mathematical paper on the $N$-spectrum, elementary observations useful for further work are presented in Section 3. In Section 4 the effect of certain digraph operations and transformations on the $N$-spectrum is studied. One family of $N$-cospectral digraphs is determined in this section. Structural similarity (i.e. values and layout of entries in the matrix) of the matrix $A A^{T}$ of some digraph with the adjacency or the signless Laplacian matrix of some multigraph, has motivated us to generalize the notion of cospectrality in Section 5 . The study of cospectrality with respect to different (multi)(di)graph matrices could be useful in finding connections between different spectral subtheories that are based on these matrices, and, what is more important, in finding new pairs of cospectral (multi)(di)graphs in particular spectral subtheory. That way, certain pairs of multigraps that are cospectral with respect to the adjacency matrix are found. The study of spectral subtheory based on the signless Laplacian matrix is currently used (see, for example, [7]), so the paper is concluded with some examples of digraphs and multigraphs whose $N$ - and $Q$-spectrum, respectively, are the same.

\section{Preliminaries}

Let $D=(V(D), E(D))$ be a digraph of order $n$ with the set of vertices $V(D)=\left\{v_{1}, v_{2}\right.$, $\left.\ldots, v_{n}\right\}$. The set of edges $E(D)$ consists of ordered pairs of vertices, and we suppose that the loops, i.e. the edges of the form $\left(v_{i}, v_{i}\right)$ are permitted, but multiple edges are not. The adjacency matrix $A=\left[a_{i j}\right]$ of $D$ is the binary matrix of order $n$, such that $a_{i j}=1$, if there is an edge from $v_{i}$ to $v_{j}$, and otherwise $a_{i j}=0$.

If $e=\left(v_{i}, v_{j}\right)$ is the edge of $D$, we say that $v_{i}$ is the initial vertex of $e$, while $v_{j}$ is the terminal vertex. The vertex $v_{j} \in V(D)$ is the out-neighbour of the vertex $v_{i} \in V(D)$ if there is the edge $\left(v_{i}, v_{j}\right) \in E(D)$. The vertex $v_{k} \in V(D)$ is the in-neighbour of the vertex $v_{i} \in V(D)$ if there is the edge $\left(v_{k}, v_{i}\right) \in E(D)$. The out-degree of vertex $v_{i}$, denoted by outdeg $g_{D}\left(v_{i}\right)$ or $d_{D}^{+}\left(v_{i}\right)$, is the number of edges of which it is the initial vertex, while the in-degree of $v_{i}$, denoted by $i n d e g_{D}\left(v_{i}\right)$ or $d_{D}^{-}\left(v_{i}\right)$, is the number of edges of which $v_{i}$ is the terminal vertex. A loop at some vertex contributes 1 to both the in-degree and the out-degree of that vertex.

Let us suppose that the edges of $D$ are ordered as $e_{1}, e_{2}, \ldots, e_{m}$. The in-incidence matrix of $D$ is the $n$ by $m$ matrix $B_{i n}=\left[b_{i j}\right]$ such that $b_{i j}=1$ if $e_{j}=\left(v_{k}, v_{i}\right)$ for some vertex $v_{k}$, and otherwise $b_{i j}=0$. The out-incidence matrix $B_{\text {out }}=\left[g_{i j}\right]$ of the digraph $D$ is the $n$ by $m$ matrix such that $g_{i j}=1$ if $e_{j}=\left(v_{i}, v_{l}\right)$ for some vertex $v_{l}$, and otherwise $g_{i j}=0$. It is a matter of routine to check that $A=B_{\text {out }} B_{\text {in }}^{T}$ holds.

The characteristic polynomial $\operatorname{det}(\lambda I-A)$ of $A$ is the characteristic polynomial of the 
digraph $D$, and the eigenvalues of $A$ are the eigenvalues of $D$. For the remaining notation and terminology related to digraphs, and also graphs, we refer the reader to [5], [2], [3], [1], [8] and [6].

In this paper we are interested in the structural characteristics of a digraph $D$ related to the spectrum of matrices $A A^{T}$ and $A^{T} A$, where $A$ is the adjacency matrix of $D$. The matrices $A A^{T}$ and $A^{T} A$ are non-negative, square and symmetric. One can easily check that these matrices are positive semi-definite (see, for example, [14]), which means that their eigenvalues are non-negative.

The entries of the matrices $A A^{T}$ and $A^{T} A$ are characterised by the following proposition (see [12]):

Proposition 2.1. The $(i, j)$-entry of the matrix $A A^{T}\left(A^{T} A\right)$ of $D$ is equal to the number of common out-neighbours (in-neighbours) of $v_{i}$ and $v_{j}$. Diagonal entries of the matrix $A A^{T}$ ( $\left.A^{T} A\right)$ represent out-degrees (in-degrees) of the vertices of $D$.

According to the previous observations, one can introduce the following notation: $N_{\text {out }}$ $=A A^{T}$ and $N_{i n}=A^{T} A$. The characteristic polynomial $\operatorname{det}\left(\lambda I-N_{i n}\right)$ of $N_{i n}$ is the $N_{i n^{-}}$ characteristic polynomial of $D$, while the characteristic polynomial $\operatorname{det}\left(\lambda I-N_{\text {out }}\right)$ of $N_{\text {out }}$ is the $N_{\text {out }}$-characteristic polynomial of $D$. Since the spectrum of $N_{\text {out }}$ and $N_{\text {in }}$ is the same (see [14]), it can be denoted by the single name - the $N$-spectrum. Therefore, the characteristic polynomials $N(x)$ of these matrices can be named the $N$-polynomials. However, we underline that through the investigation we mainly considered $N_{\text {out }}(D)$ matrix of $D$, whose spectrum is denoted by $\eta_{1} \geq \eta_{2} \geq \ldots \geq \eta_{n}$. The $N$-spectral radius $\rho_{N}(D)$ of $D$ is defined to be the spectral radius of $N_{\text {out }}(D)$, and similarly $N_{\text {in }}(D)$.

Remark 2.2. For the $N$-spectrum $\eta_{1}, \eta_{2}, \ldots, \eta_{n}$ of a digraph $D$ with $m$ edges the following holds:

- The numbers $\eta_{1}, \eta_{2}, \ldots, \eta_{n}$ are real and non-negative,

- $\eta_{1}+\eta_{2}+\ldots+\eta_{n}=m$,

- $D$ is consisted of only isolated vertices if and only if $\eta_{1}=\eta_{2}=\ldots=\eta_{n}=0$.

\section{Some basic results}

In this section we give some elementary results that we will use in the subsequent sections.

Let us remind you that a digraph $D$ is $r$-regular if the in-degree and the out-degree of each its vertex are equal to $r$. By use of the basic combinatorial principles for counting one can easily check that the row sum for each row of the matrix $N_{\text {out }}(D)$ is equal to $r+r(r-1)=r^{2}$. Now, we can prove the following lemma:

Lemma 3.1. $N$-spectral radius $\rho_{N}(D)$ of a r-regular digraph $D$ of order $n$ is $r^{2}$.

Proof. Since $N_{\text {out }}(D)$ is the square, non-negative matrix with equal row sums, according to Theorem of Frobenius (see [4]) the spectral radius of this matrix is $r^{2}$.

Remark 3.2. The eigenvector that corresponds to the $N$-eigenvalue $r^{2}$ of a $r$-regular digraph $D$ is all-1 vector. 
Example 3.3. The complete digraph of order $n$ is the digraph $\overleftrightarrow{K}_{n}$ in which for each pair of vertices there is an edge, including a loop at each vertex. The $N$-characteristic polynomial of this digraph is:

$$
N_{\overleftrightarrow{K_{n}}}(x)=\left(x-n^{2}\right) x^{n-1},
$$

and thus its $N$-spectrum is: $n^{2},[0]^{n-1}$.

Here, and in the further text, an eigenvalue $\eta$ of the multiplicity $k$ is denoted by $[\eta]^{k}$.

Let us now consider connected digraphs whose vertices do not have the common outneighbours. If $D=(V(D), E(D))$ is such a digraph, then $\operatorname{indeg}_{D}\left(v_{i}\right) \leq 1$ must hold for each vertex $v_{i} \in V(D)$.

Let us remind you that a rooted oriented tree, briefly rooted tree, is an oriented tree with a specific vertex $v_{1}$, called the root, such that for every other vertex $v_{j}$ the path connecting $v_{1}$ to $v_{j}$ is a directed path from $v_{1}$ to $v_{j}$. This means that $D$ is connected, $\operatorname{indeg}\left(v_{1}\right)=0$ and $i \operatorname{deg} g_{D}\left(v_{i}\right)=1$ for every other vertex $v_{i}$ of $D$, and vice versa according to Theorem 15.2 from [1]. It is obvious that vertices of a rooted tree do not have the common outneighbours.

If in a digraph $D$ whose vertices do not have the common out-neighbours there are at least two vertices such that their in-degrees are equal to $0, D$ would not have been connected, i.e. $D$ would consist of at least two connected components.

Since in a rooted tree there is unique vertex $v_{1}$ such that $\operatorname{indeg}\left(v_{1}\right)=0$, one can add one extra edge to obtain a digraph where there is no pair of vertices with common outneighbours. We distinguish two possibilities: this extra edge is a loop at $v_{1}$, i.e. $\left(v_{1}, v_{1}\right)$ or it is an edge $\left(v_{x}, v_{1}\right)$, for exactly one vertex $v_{x}$ of a rooted tree. Hence, we can say that a resulting digraph is a unicyclic digraph derived from a rooted tree (Figure 1).
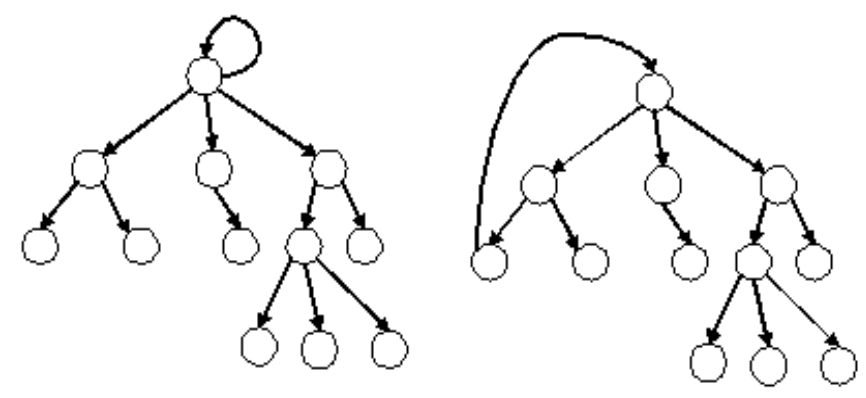

Figure 1: Unicyclic digraphs whose vertices do not have the common out-neighbours

That way, the following proposition is proved:

Proposition 3.4. $D$ is a connected digraph whose vertices do not have the common outneighbours if and only if it is a rooted tree or a unicyclic digraph that can be derived from a rooted tree.

Remark 3.5. Since the matrix $N_{\text {out }}(D)$ of a connected digraph $D$ such that there is no pair of vertices with the common out-neighbours in $D$ is the diagonal matrix of vertex degrees, the $N$-spectrum of $D$ is: $\operatorname{outdeg}_{D}\left(v_{1}\right), \operatorname{outdeg}_{D}\left(v_{2}\right), \ldots$, outdeg $_{D}\left(v_{n}\right)$. 
Remark 3.6. The converse digraph $\operatorname{Conv}(D)$ of a digraph $D$ is obtained by reversing the direction of each edge of $D$ (see [2]). So, a digraph whose vertices do not have the common in-neighbours is the converse digraph of a rooted tree or of a unicyclic digraph that can be derived from a rooted tree.

Example 3.7. The $N$-characteristic polynomial of a rooted tree $D$ is:

$$
N_{D}(x)=x^{l} \prod_{v_{i} \in U(D)}\left(x-\operatorname{outdeg}\left(v_{i}\right)\right),
$$

where $l$ is the number of vertices $v_{x}$ such that $\operatorname{outdeg}_{D}\left(v_{x}\right)=0$, while $U(D) \subset V(D)$ is the set of vertices whose out-degree is at least 1 .

The digraph $\overrightarrow{P_{n}}$ is the special case of a rooted oriented tree. If $V\left(\overrightarrow{P_{n}}\right)=\left\{v_{1}, v_{2}, \ldots\right.$, $\left.v_{n}\right\}$ is the set of vertices of this digraph, then its set of edges consists of the pairs of vertices $\left(v_{i}, v_{i+1}\right)$, for $i=1,2, \ldots, n-1$. The $N$-characteristic polynomial of $\overrightarrow{P_{n}}$ is:

$$
N_{P_{n}}(x)=x(x-1)^{n-1} .
$$

1-regular digraph $\vec{C}_{n}$ is the special case of a unicyclic digraph derived from a rooted tree. Its $N$-characteristic polynomial is:

$$
N_{\overrightarrow{C_{n}}}(x)=(x-1)^{n} .
$$

\section{Some digraph operations and transformations}

We open this section with the result related to the $N$-spectrum of the complement of a given regular digraph.

The complement $D^{C}=\left(V\left(D^{C}\right), E\left(D^{C}\right)\right)$ of a digraph $D=(V(D), E(D))$ has the vertex set $V\left(D^{C}\right)=V(D)$ and $e \in E\left(D^{C}\right)$ if and only if $e \notin E(D)$. Also, there is a loop at vertex $v_{i}$ in $D^{C}$ if and only if there is no loop at $v_{i}$ in $D$. Similarly to the proof of Theorem 2.1.2 from [6] for regular graphs we can prove the following:

Proposition 4.1. If the $N$-eigenvalues of a $r$-regular digraph $D$ of order $n$ are $\eta_{i}(D), i=$ $1,2, \ldots, n$, then the $N$-eigenvalues of $D^{C}$ are $\eta_{1}\left(D^{C}\right)=(n-r)^{2}$ and $\eta_{i}\left(D^{C}\right)=\eta_{i}(D)$, $i=2,3, \ldots, n$.

Proof. If $A_{D}$ is the adjacency matrix of $D$ and $J$ is all-1 matrix, we find:

$$
N_{\text {out }}\left(D^{C}\right)=J^{2}-A_{D} J-J A_{D}^{T}+A_{D} A_{D}^{T}=(n-2 r) J+N_{\text {out }}(D),
$$

because the row sum for each row of $A_{D}$ is equal to $r$.

Let us denote by $D^{\prime}$ the digraph obtained from a connected digraph $D$ by deleting the edge $\left(v_{i}, v_{j}\right)$. Then we have: $N_{\text {out }}(D)=N_{\text {out }}\left(D^{\prime}\right)+M$. Here, $M=\left[m_{p q}\right]$ is the square matrix of order $n$ such that $m_{i i}=1$ and $m_{i l}=m_{l i}=1$ for each pair of vertices $v_{i}, v_{l}$ such that $\left(v_{i}, v_{j}\right),\left(v_{l}, v_{j}\right) \in E(D)$, where $l \in\{1,2, \ldots, n\} \backslash\{i\}$.

Theorem 4.2. (Interlacing theorem - edge version) Let $D$ be a connected digraph of order $n$ whose $N$-spectrum is $\eta_{1}(D) \geq \eta_{2}(D) \geq \cdots \geq \eta_{n}(D)$, and there is at least one 
vertex $v_{j}$ in $D$ such that indeg $g_{D}\left(v_{j}\right)=1$. Let $D^{\prime}$ be a digraph obtained from $D$ by deleting an edge $\left(v_{i}, v_{j}\right)$. If the $N$-eigenvalues of $D^{\prime}$ are $\eta_{1}\left(D^{\prime}\right) \geq \eta_{2}\left(D^{\prime}\right) \geq \cdots \geq \eta_{n}\left(D^{\prime}\right)$, then

$$
\eta_{1}(D) \geq \eta_{1}\left(D^{\prime}\right) \geq \eta_{2}(D) \geq \eta_{2}\left(D^{\prime}\right) \geq \ldots \eta_{n}(D) \geq \eta_{n}\left(D^{\prime}\right) \geq 0 .
$$

Proof. Since the spectrum of the matrix $M$ consists of [1] and $[0]^{n-1}$, the proof follows from Courant-Weyl inequalities (see, for example [6]).

Remark 4.3. By considering $N_{i n}$ matrix of a digraph, one can prove that the previously given Interlacing theorem holds also for a connected digraph $D$ in which there is at least one vertex $v_{j}$ such that outdeg $_{D}\left(v_{j}\right)=1$, and for its subdigraph $D^{\prime}$ obtained from $D$ by deleting an edge $\left(v_{j}, v_{i}\right)$, for some vertex $v_{i}$.

In general case, such the $N$-eigenvalue interlacing does not hold. Namely, we have the following example.

Example 4.4. For the digraph $D$ that is depicted on Figure 2 and the digraph $D^{\prime}$ that is obtained from $D$ by deleting the edge $(1,3)$, the $N$-interlacing property holds, i.e. for the $N$-spectra of these digraphs we have the following inequalities: $4.390 \geq 3.879 \geq 1.838 \geq$ $1.653 \geq 1 \geq 1 \geq 0.544 \geq 0.468 \geq 0.228 \geq 0$.

On the other hand, the $N$-eigenvalues of the digraphs $D_{1}$ (Figure 2) and $D_{1}^{\prime}$, that is obtained from $D_{1}$ by deleting the edge $(1,3)$, are $5.303 \geq 1.697 \geq 1 \geq 1 \geq 0$, and similarly $4.115 \geq 1.764 \geq 1 \geq 1 \geq 0.139$, so the $N$-interlacing property does not hold in this case.
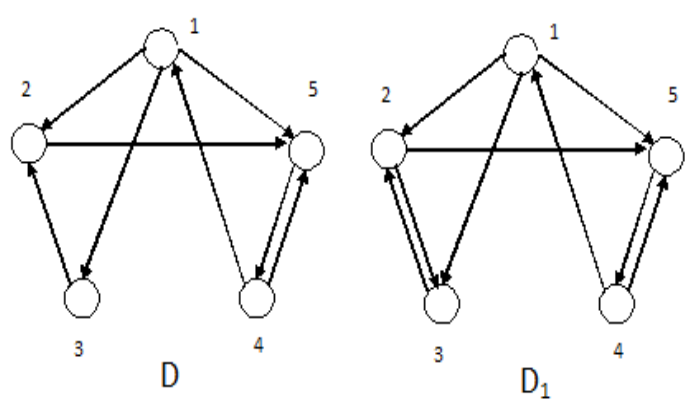

Figure 2: Digraphs $D$ and $D_{1}$ from Example 4.4

Now, we will consider a digraph $D^{*}$ obtained from a connected digraph $D$ by adding a pendant edge at the vertex $v_{i}$ of $D$ (i.e. an edge of the form $\left(v_{x}, v_{i}\right)$ such that indeg $g_{D^{*}}\left(v_{x}\right)$ $=0$ and $\operatorname{outdeg}_{D^{*}}\left(v_{x}\right)=1$, or an edge of the form $\left(v_{i}, v_{x}\right)$ such that $\operatorname{indeg}_{D^{*}}\left(v_{x}\right)=1$ and outdeg $\left._{D^{*}}\left(v_{x}\right)=0\right)$.

The following statement obviously holds.

Proposition 4.5. Let $D^{*}$ denotes a digraph obtained from a connected digraph $D$ of order $n$ by adding a pendant edge $\left(v_{n+1}, v_{i}\right)$ at the vertex $v_{i}$ such that indeg $g_{D}\left(v_{i}\right)=0$. Then the $N$-characteristic polynomial of $D^{*}$ is: $N_{D^{*}}(x)=(x-1) N_{D}(x)$.

Let us denote by $D_{v_{k}}$ a digraph obtained from a digraph $D$ by deleting the vertex $v_{k}$, and let $\mu_{D}\left(v_{i}, v_{j}\right)=1$, if $\left(v_{i}, v_{j}\right) \in E(D)$, and otherwise $\mu_{D}\left(v_{i}, v_{j}\right)=0$, for $i, j \in$ $\{1,2, \ldots, n\}$. 
Definition 4.6. The digraph $D_{\left(v_{k}, v_{i}\right)}^{\text {out }}$ is the out- $\left(v_{k}, v_{i}\right)$-shrinking of $D$ if for the edge $\left(v_{k}, v_{i}\right)$ in $E(D), V\left(D_{\left(v_{k}, v_{i}\right)}^{\text {out }}\right)=V\left(D_{v_{k}}\right)$ and

$$
E\left(D_{\left(v_{k}, v_{i}\right)}^{\text {out }}\right)=E\left(D_{v_{k}}\right) \cup\left\{\left(v_{j}, v_{i}\right) \mid \mu_{D}\left(v_{j}, v_{k}\right)=1 \text {, for each } j \neq k\right\} .
$$

It is obvious that $D_{\left(v_{k}, v_{i}\right)}^{\text {out }}$ is a multidigraph in general case, and that if $\operatorname{indeg_{D}}\left(v_{i}\right)=1$ then the matrix $N_{\text {out }}\left(D_{\left(v_{k}, v_{i}\right)}^{\text {out }}\right)$ equals the matrix obtained from $N_{\text {out }}(D)$ by deleting the $k$-th row and the $k$-th column.

Theorem 4.7. Let $D^{*}$ denotes a digraph obtained from a connected digraph $D$ of order $n$ by adding the pendant edge $\left(v_{j}, v_{i}\right)$ at the vertex $v_{i}$ such that $\left(v_{k}, v_{i}\right) \in E(D)$ and $\operatorname{indeg}_{D}\left(v_{i}\right)=1$. Then

$$
N_{D^{*}}(x)=(x-1) N_{D}(x)-N_{D_{\left(v_{k}, v_{i}\right)}^{o u t}}(x),
$$

where $N_{D_{\left(v_{k}, v_{i}\right)}^{\text {out }}}(x)$ is the $N$-characteristic polynomial of the digraph $D_{\left(v_{k}, v_{i}\right)}^{\text {out }}$ that is the out- $\left(v_{k}, v_{i}\right)$-shrinking of a digraph $D$.

Proof. Since indeg $_{D^{*}}\left(v_{i}\right)=2$, we have

$$
N_{\text {out }}\left(D^{*}\right)=\left(\begin{array}{cc}
N_{\text {out }}(D) & r \\
r^{T} & 1
\end{array}\right)_{(n+1) \times(n+1)},
$$

where $r=(0, \ldots, 0, \underbrace{1}_{k}, 0, \ldots, 0)^{T}$ is the vector of order $n$. The only no null coordinate of the vector $r$ corresponds to the common out-neighbour of $v_{k}$ and $v_{j}$. By expanding the determinant of the matrix $x I-N_{\text {out }}\left(D^{*}\right)$ by the last row we get:

$$
N_{D^{*}}(x)=\operatorname{det}\left(x I-N_{\text {out }}\left(D^{*}\right)\right)=(x-1) N_{D}(x)+(-1)^{(n+1)+k} \cdot \operatorname{det}(M \mid r),
$$

where the matrix $M$ is obtained from $x I-N_{\text {out }}(D)$ by deleting the $k$-th column. Now, by expanding the determinant of the matrix $(M \mid r)$ by the last column, we have:

$$
\begin{aligned}
N_{D^{*}}(x)= & (x-1) N_{D}(x)+(-1)^{(n+1)+k}(-1)^{k+n} \operatorname{det}\left(x I-M^{\prime}\right)= \\
& (x-1) N_{D}(x)-\operatorname{det}\left(x I-M^{\prime}\right),
\end{aligned}
$$

where $M^{\prime}$ is obtained from the matix $N_{\text {out }}(D)$ by deleting the $k$-th row and $k$-th column.

The line digraph $L(D)$ of a digraph $D$ (see, for example [5]) is the digraph whose vertices are the edges $e_{1}, e_{2}, \ldots, e_{m}$ of $D$ such that there is an edge from $e_{i}$ to $e_{j}$ in $L(D)$ if and only if the terminal vertex of $e_{i}$ equals the initial vertex of $e_{j}$ in $D$. If an edge $e_{p}$ is a loop at some vertex of $D$, then it becomes a loop at $e_{p}$ in $L(D)$.

Some results on adjacency spectra and energies of iterated line graphs are exposed in [13]. On the similar way, we can define iterated line digraphs. If $D=L^{0}(D)$ is a digraph and $L(D)=L^{1}(D)$ is its line digraph, then $L^{k}(D), k=2,3, \ldots$ defined recursively by the formula $L^{k}(D)=L\left(L^{k-1}(D)\right)$ are the iterated line digraphs of $D$. The line digraph of an $r$-regular digraph is also $r$-regular digraph. More precisely, the line digraph $L^{1}(D)$ of an $r$ regular digraph $D$ of order $n$ is the $r_{1}=r$-regular digraph of order $n_{1}=n r$. Consequently, $L^{k}(D), k=2,3, \ldots$ is the $r_{k}=r$-regular digraph of order $n_{k}=r n_{k-1}=r^{k} n$, where $n_{k-1}$ is the order of the digraph $L^{k-1}(D)$. 
Theorem 4.8. The $N$-eigenvalues of the line digraph $L(D)$ of a $r$-regular digraph $D$ are: $\left[r^{2}\right]^{n},[0]^{(r-1) n}$.

Proof. We will determine the $N$-characteristic polynomial $N_{L(D)}$ of $L(D)$ related to the $N_{\text {out }}(L(D))$ matrix.

As $L=B_{i n}^{T} B_{\text {out }}$ is the adjacency matrix of the line digraph $L(D)$ of $D$ (see [5]), where $B_{\text {in }}$ and $B_{\text {out }}$ are the in-incidence matrix and the out-incidence matrix of $D$, respectively, we find: $N_{\text {out }}(L(D))=r B_{i n}^{T} B_{\text {in }}$. Here, we have that the diagonal matrix whose entries are the out(in)-degrees of vertices in $D$ is: $\Delta=r I=B_{\text {in }} B_{\text {in }}^{T}=B_{\text {out }} B_{\text {out }}^{T}$.

According to Lemma 8.2.3. from [10] we get:

$$
\operatorname{det}\left(I-B_{i n} B_{i n}^{T}\right)=\operatorname{det}\left(I-B_{i n}^{T} B_{i n}\right),
$$

i.e.

$$
\operatorname{det}\left(I_{n}-x^{-1} r I_{n}\right)=\operatorname{det}\left(I_{m}-x^{-1} \frac{1}{r} N_{\text {out }}(L(D))\right) .
$$

Furthermore we have:

$$
x^{m-n} \operatorname{det}\left(x I_{n}-r I_{n}\right)=\operatorname{det}\left(x I_{m}-\frac{1}{r} N_{\text {out }}(L(D))\right),
$$

and also

$$
\operatorname{det}\left((x-r+1) I_{n}-I_{n}\right)=x^{n-m} \frac{1}{r^{m}} \operatorname{det}\left(r x I_{m}-N_{\text {out }}(L(D))\right) .
$$

According to (3.1) we find:

$$
N_{\vec{C}_{n}}(x-r+1)=x^{n-m} \frac{1}{r^{m}} N_{L(D)}(r x),
$$

i.e.

$$
N_{L(D)}(x)=x^{m-n}\left(x-r^{2}\right)^{n},
$$

and the proof follows.

Therefore the $N$-spectrum of the line digraph $L^{k}(D)$ of a $r$-regular digraph $D$ of order $n$ consists of $\left[r^{2}\right]^{n_{k}}=\left[r^{2}\right]^{n r^{k}}$ and $[0]^{(r-1) n_{k}}=[0]^{(r-1) r^{k} n}$, and hence we have the following corollary:

Corollary 4.9. Let $D_{1}$ and $D_{2}$ be two r-regular digraphs of order $n$ (not necessary $N$ cospectral). Then for all $k \geq 1$ digraphs $L^{k}\left(D_{1}\right)$ and $L^{k}\left(D_{2}\right)$ are $N$-cospectral.

This way, we found a family of $N$-cospectral mates (i.e. the digraphs whose $N$-spectra are the same). We will continue examination of cospectrality in the next section.

\section{Cospectrality relation}

Let $\mathcal{D}_{M}^{n}$ be the set of (multi)(di)graphs $D$ of order $n$ with the associated spectrum $\sigma_{M}(D)$ related to some (multi)(di)graph matrix $M$. Let us introduce the relation $\rho \subseteq \mathcal{D}_{M_{1}}^{n} \times \mathcal{D}_{M_{2}}^{n}$ between sets $\mathcal{D}_{M_{1}}^{n}$ and $\mathcal{D}_{M_{2}}^{n}$, for some (multi)(di)graph matrices $M_{1}$ and $M_{2}$ in the following way: we say that the (multi)(di)graph $D_{1}$ is in the relation $\rho$ with the (multi)(di)graph $D_{2}$, i.e. $D_{1} \rho D_{2}$ if and only if $\sigma_{M_{1}}\left(D_{1}\right)=\sigma_{M_{2}}\left(D_{2}\right)$. So, the relation $\rho$ is the cospectrality relation, while $D_{1}$ and $D_{2}$ form an $\left(M_{1}, M_{2}\right)$-cospectral mate. That way, we can generalize the notion of cospectrality: 
Definition 5.1. Let $M_{1}$ and $M_{2}$ be some (multi)(di)graph matrices. If the (multi)(di)graph $D_{1} \in \mathcal{D}_{M_{1}}^{n}$ is in the cospectrality relation $\rho$ with the (multi)(di)graph $D_{2} \in \mathcal{D}_{M_{2}}^{n}$, i.e. the $M_{1}$-spectrum of a (multi)(di)graph $D_{1}$ is equal to the $M_{2}$-spectrum of a (multi)(di)graph $D_{2}$, then $D_{1}$ and $D_{2}$ are called $\left(M_{1}, M_{2}\right)$-cospectral (multi)(di)graphs.

It is obvious that $\rho$ is the equivalence relation on the set $\mathcal{D}_{M}^{n}$, in which case (multi)(di)graphs $D_{1}$ and $D_{2}$ such that $D_{1} \rho D_{2}$ are $M$-cospectral. As a result of the composition of the cospectrality relations, one can get some new pairs of cospectral (multi)(di)graphs, as follows.

Let us consider the set $\mathcal{D}_{N}^{n}$ of digraphs $D$ of order $n$ with the associated $N$-spectrum $\sigma_{N}(D)$. Clearly, $N$ is related to $N_{\text {out }}$ or $N_{\text {in }}$ matrix of a digraph. Let us denote by $\mathcal{G}_{A^{+}}^{n}$ and $\mathcal{G}_{A^{-}}^{n}$ the sets of out-multigraphs and in-multigraphs, respectively with the corresponding adjacency spectra. The in-multigraph $M_{D}^{-} \in \mathcal{G}_{A^{-}}^{n}$ and the out-multigraph $M_{D}^{+} \in \mathcal{G}_{A^{+}}^{n}$ are associated to a digraph $D \in \mathcal{D}_{N}^{n}$ in the following way:

Definition 5.2. The in-multigraph $M_{D}^{-}=\left(V\left(M_{D}^{-}\right), E\left(M_{D}^{-}\right)\right)$of a digraph $D$ is the multigraph such that $V\left(M_{D}^{-}\right)=V(D),\left\{v_{i}, v_{j}\right\} \in E\left(M_{D}^{-}\right)$if and only if there is a vertex $v_{k} \in V(D)$ such that $\left(v_{k}, v_{i}\right),\left(v_{k}, v_{j}\right) \in E(D)$, and for each edge $\left(v_{k}, v_{i}\right)$ in $D$ there is a loop at $v_{i}$ in $M_{D}^{-}$.

Definition 5.3. The out-multigraph $M_{D}^{+}=\left(V\left(M_{D}^{+}\right), E\left(M_{D}^{+}\right)\right)$of a digraph $D$ is the multigraph such that $V\left(M_{D}^{+}\right)=V(D),\left\{v_{i}, v_{j}\right\} \in E\left(M_{D}^{+}\right)$if and only if there is a vertex $v_{k}$ such that $\left(v_{i}, v_{k}\right),\left(v_{j}, v_{k}\right) \in E(D)$, and for each edge $\left(v_{i}, v_{k}\right)$ in $D$ there is a loop at $v_{i}$ in $M_{D}^{+}$.

According to the previous definitions, one can notice the cospectrality relation, say $\rho_{-}$, between sets $\mathcal{G}_{A^{-}}^{n}$ and $\mathcal{D}_{N}^{n}$, and similarly the cospectrality relation, say $\rho_{+}$, between sets $\mathcal{D}_{N}^{n}$ and $\mathcal{G}_{A^{+}}^{n}$. As the result of the composition of relations $\rho_{+}$and $\rho_{-}$the pairs of $A$-cospectral multigraphs $M_{D}^{-}$and $M_{D}^{+}$are getting. That way we have:

Theorem 5.4. Multigraphs $M_{D}^{-}$and $M_{D}^{+}$are A-cospectral.

So, the exposed construction is a way for obtaining new pairs of cospectral and not necessarily isomorphic multigraphs.

Example 5.5. The adjacency matrix of the in-multigraph $M_{D}^{-}$, and similarly the outmultigraph $M_{D}^{+}$, that is associated to the digraph $D$ (which is depicted on Figure 3 ) is:

$$
A\left(M_{D}^{-}\right)=N_{\text {in }}(D)=\left(\begin{array}{cccc}
2 & 0 & 2 & 0 \\
0 & 1 & 1 & 0 \\
2 & 1 & 3 & 0 \\
0 & 0 & 0 & 1
\end{array}\right) \text {, and } A\left(M_{D}^{+}\right)=N_{\text {out }}(D)=\left(\begin{array}{cccc}
2 & 1 & 0 & 1 \\
1 & 2 & 0 & 2 \\
0 & 0 & 1 & 0 \\
1 & 2 & 0 & 2
\end{array}\right) \text {. }
$$

Remark 5.6. Multigraphs $M_{D}^{-}$and $M_{D}^{+}$associated to a digraph $D$ are simple graphs only in the case when digraph $D$ is a set of isolated vertices. If we permit existence of single loops (i.e. loops of multiplicity one) in a simple graph, the primary digraph $D$ can be $\overrightarrow{C_{n}}$ or $\vec{P}_{n}$. In this case, multigraphs $M_{D}^{-}$and $M_{D}^{+}$are the sets of isolated loops or the disjoint unions of isolated loops and a single isolated vertex, and therefore $M_{D}^{-}$and $M_{D}^{+}$are not only $A$-cospectral but also isomorphic. 

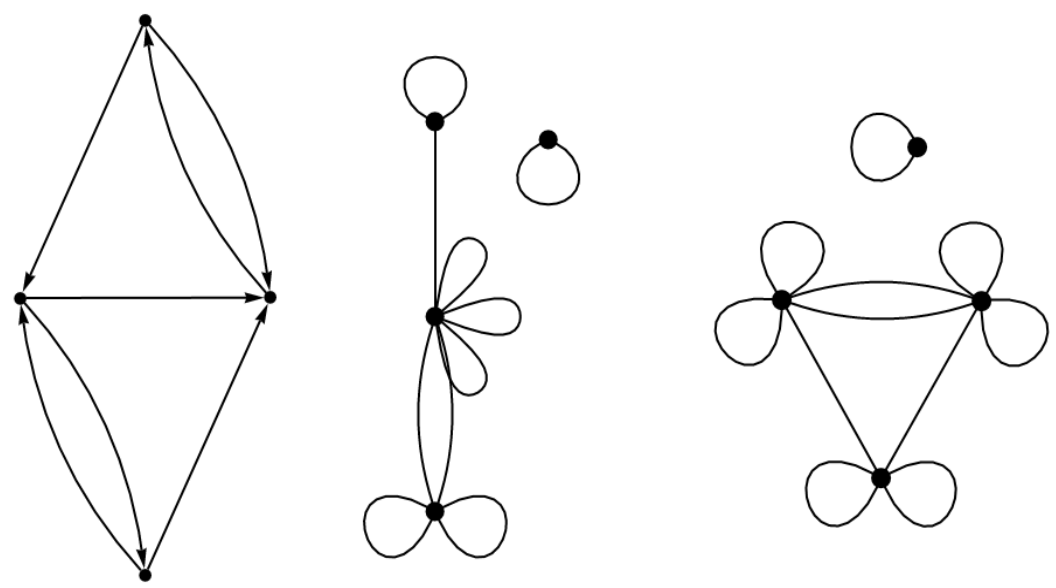

Figure 3: Digraph $D$ from Example 5.5 and associated multigraphs $M_{D}^{-}$and $M_{D}^{+}$, respectively

There are many examples where the multigraphs $M_{D}^{-}$and $M_{D}^{+}$associated to a given digraph $D$ are isomorphic, so the investigation of such multigraphs can be the subject of future research. If a primary digraph $D$ is such that if $\left(v_{i}, v_{j}\right) \in E(D)$ then also $\left(v_{j}, v_{i}\right) \in$ $E(D)$, for all $v_{i}, v_{j} \in V(D)$, it is obvious that the associated multigraphs $M_{D}^{-}$and $M_{D}^{+}$ will be isomorphic. We also have:

Proposition 5.7. Multigraphs $M_{D}^{-}$and $M_{D}^{+}$associated to a digraph $D$ of prime order, $n>2$, with circulant adjacency matrix are isomorphic.

Proof. Since $N_{\text {in }}(D)$ and $N_{\text {out }}(D)$ are circulant matrices with the same eigenvalues, according to Theorem 1 from [9] they are permutationally similar.

For an integer $n \geq 2$ and a set $S \subseteq\{1,2, \ldots, n-1\}$ the circulant digraph $C_{n}(S)$ is a digraph such that $V\left(C_{n}(S)\right)=\{1,2, \ldots, n\}$ and $E\left(C_{n}(S)\right)=\{(i, i+j(\bmod n)): 1 \leq$ $i \leq n, j \in S\}$. Circulant digraphs are of great interest in the graph and digraph theory and their applications (see [2]).

Proposition 5.8. Multigraphs $M_{D}^{-}$and $M_{D}^{+}$associated to a circulant digraph $C_{n}(S)$ are isomorphic.

Proof. Since the converse digraph $\operatorname{Conv}\left(C_{n}(S)\right)$ of $C_{n}(S)$ is isomorphic to $C_{n}(S)$ (according to Proposition 2.14.1 from [2]) and since $N_{\text {in }}\left(C_{n}(S)\right)=N_{\text {out }}\left(\operatorname{Conv}\left(C_{n}(S)\right)\right)$, and similarly $N_{\text {out }}\left(C_{n}(S)\right)=N_{\text {in }}\left(\operatorname{Conv}\left(C_{n}(S)\right)\right)$, the proof follows.

Example 5.9. The matrix $N_{\text {out }}(D)$ of the 2-regular digraph $D$ that is depicted on Figure 4 structurally corresponds to the signless Laplacian matrix $Q(M)$ of the 2-regular graph $M$, also depicted on Figure 4, i.e.

$$
N_{\text {out }}(D)=\left(\begin{array}{llll}
2 & 1 & 1 & 0 \\
1 & 2 & 0 & 1 \\
1 & 0 & 2 & 1 \\
0 & 1 & 1 & 2
\end{array}\right)=\left(\begin{array}{llll}
0 & 1 & 1 & 0 \\
1 & 0 & 0 & 1 \\
1 & 0 & 0 & 1 \\
0 & 1 & 1 & 0
\end{array}\right)+\left(\begin{array}{llll}
2 & 0 & 0 & 0 \\
0 & 2 & 0 & 0 \\
0 & 0 & 2 & 0 \\
0 & 0 & 0 & 2
\end{array}\right)=Q(M)
$$


That way, one can notice the cospectrality relation $\rho \subseteq \mathcal{D}_{N}^{n} \times \mathcal{G}_{Q}^{n}$ between set $\mathcal{D}_{N}^{n}$ of digraphs $D$ of order $n$ with the associated $N$-spectrum $\sigma_{N}(D)$ and the set $\mathcal{G}_{Q}^{n}$ of multigraphs $M$ of order $n$ with the associated $Q$-spectrum $\sigma_{Q}(M)$.

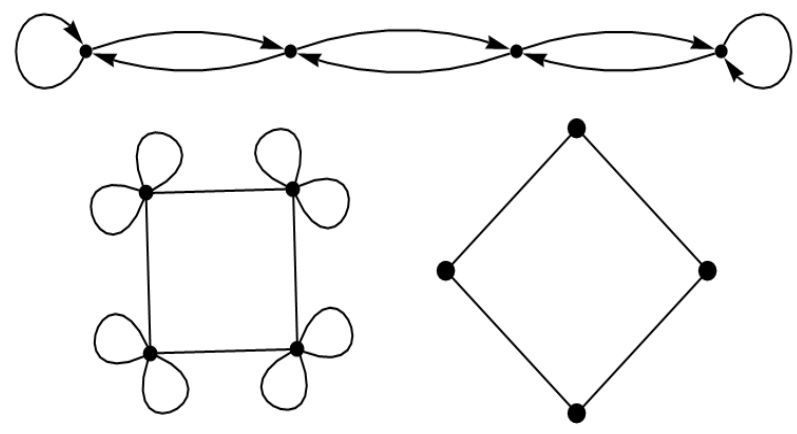

Figure 4: Triplet of $(N, A, Q)$-cospectral digraph $D$, multigraph $M_{D}^{-}=M_{D}^{+}$and graph $M$, respectively

This one and similar examples have motivated us to examine some new $(N, Q)$-cospectral mates. Furthermore, the multigraph $M$ that makes $(Q, N)$-cospectral mate with a given digraph $D$ can be used in determining some isomorphic multigraphs $M_{D}^{-}$and $M_{D}^{+}$, as follows:

Proposition 5.10. Let $D$ be a connected r-regular digraph of order $n$. If $N_{\text {out }}(D)=$ $Q(M)$ holds for some multigraph $M$, then $r=0$ or $r=2$.

Proof. We have $N_{\text {out }}(D)=r I+C$, where row sum of $C$ is $r(r-1)$ for each row.

If $N_{\text {out }}(D)$ is the signless Laplacian matrix of some multigraph without loops, then $r=r(r-1)$ holds, which implies $r=0$ or $r=2$. On the other hand, if $N_{\text {out }}(D)$ is the signless Laplacian matrix of a multigraph with loops, then the number of loops at some vertex is $(r-(r-1) r) / 2$, which means that $r=0$ or $r=2$.

Remark 5.11. The statement from the previous proposition also holds in the case of the matrix $N_{i n}(D)$. Beside that, the multigraph $M$ is the connected $r$-regular multigraph without loops. Therefore, we conclude that multigraphs $M_{D}^{-}$and $M_{D}^{+}$associated to some 2regular digraph $D$ are isomorphic.

In order to examine $(N, Q)$-cospectrality, we will introduce some binary digraph operations. Still, according to the nature and the mutual relationships between entries of matrices $N_{\text {out }}(D)$ and $Q(M)$ of some digraph $D$ and some multigraph $M$, respectively, one can suspect poor variety in terms of the structure and the order (i.e. number of vertices) of the $(N, Q)$-cospectral mates (that could be obtained by direct comparing of these matrices).

Let $D_{1}=\left(V\left(D_{1}\right), E\left(D_{1}\right)\right)$ and $D_{2}=\left(V\left(D_{2}\right), E\left(D_{2}\right)\right)$ be two disjoint digraphs (i.e. digraphs with no common vertices nor edges).

Definition 5.12. The out-join $D_{1} \nabla_{\text {out }} D_{2}$ of two disjoint digraphs $D_{1}=\left(V\left(D_{1}\right), E\left(D_{1}\right)\right)$ and $D_{2}=\left(V\left(D_{2}\right), E\left(D_{2}\right)\right)$ is the digraph $D=(V(D), E(D))$ such that $V(D)=$ 
$V\left(D_{1}\right) \cup V\left(D_{2}\right)$ and $E(D)=E\left(D_{1}\right) \cup E\left(D_{2}\right) \cup\left\{(u, v) \mid u \in V\left(D_{1}\right), v \in V\left(D_{2}\right)\right\}$, for each $u \in V\left(D_{1}\right)$ and $v \in V\left(D_{2}\right)$.

It is obvious that this digraph operation is not commutative, i.e. $D_{1} \nabla_{\text {out }} D_{2} \neq D_{2} \nabla_{\text {out }}$ $D_{1} . N_{\text {out }}(D)$ matrix of the digraph $D$ which is obtained by out-joining is:

$$
\begin{gathered}
N_{\text {out }}(D)=N_{\text {out }}\left(D_{1} \nabla_{\text {out }} D_{2}\right)=\left(\begin{array}{cc}
A_{1} & J \\
O & A_{2}
\end{array}\right)\left(\begin{array}{cc}
A_{1}^{T} & O^{T} \\
J^{T} & A_{2}^{T}
\end{array}\right)= \\
\left(\begin{array}{cc}
N_{\text {out }}\left(D_{1}\right)+J J^{T} & \left(A_{2} J^{T}\right)^{T} \\
A_{2} J^{T} & N_{\text {out }}\left(D_{2}\right)
\end{array}\right),
\end{gathered}
$$

where $A_{1}$ and $A_{2}$ are the adjacency matrices of digraphs $D_{1}$ and $D_{2}$, respectively, while $J$ is all-1 matrix. Each entry of the $j$-th row of the matrix $A_{2} J^{T}$ is equal to outdeg $g_{D_{2}}\left(u_{j}\right)$, where $u_{j} \in V\left(D_{2}\right)$.

In the same way one can define:

Definition 5.13. The in-join $D_{1} \nabla_{i n} D_{2}$ of two disjoint digraphs $D_{1}=\left(V\left(D_{1}\right), E\left(D_{1}\right)\right)$ and $D_{2}=\left(V\left(D_{2}\right), E\left(D_{2}\right)\right)$ is the digraph $D=(V(D), E(D))$ such that $V(D)=$ $V\left(D_{1}\right) \cup V\left(D_{2}\right)$ and $E(D)=E\left(D_{1}\right) \cup E\left(D_{2}\right) \cup\left\{(v, u) \mid v \in V\left(D_{2}\right), u \in V\left(D_{1}\right)\right\}$, for each $u \in V\left(D_{1}\right)$ and $v \in V\left(D_{2}\right)$.

Definition 5.14. The join $D_{1} \nabla D_{2}$ of two disjoint digraphs $D_{1}=\left(V\left(D_{1}\right), E\left(D_{1}\right)\right)$ and $D_{2}=\left(V\left(D_{2}\right), E\left(D_{2}\right)\right)$ is the digraph $D$ with the vertex set $V(D)=V\left(D_{1}\right) \cup V\left(D_{2}\right)$, whose set of edges is $E(D)=\left(E\left(D_{1} \nabla_{\text {out }} D_{2}\right) \cup E\left(D_{1} \nabla_{\text {in }} D_{2}\right)\right) \backslash\left(E\left(D_{1}\right) \cup E\left(D_{2}\right)\right)$.

Proposition 5.15. Let $D=D_{1} \nabla_{\text {out }} D_{2}$ be the digraph obtained by out-joining two connected disjoint digraphs $D_{1}$ and $D_{2}$ of orders $n_{1}$ and $n_{2}$, respectively. If $N_{\text {out }}(D)=$ $Q(M)$ holds for some multigraph $M$, then:

1. $D_{1}$ is an isolated vertex, while $D_{2}$ is a unicyclic digraph derived from a rooted tree.

2. $D_{1}=\overleftrightarrow{K}_{1}$, while $D_{2}$ is a rooted tree;

3. $D_{2}$ is an isolated vertex, and:

(a) if $n_{1}=1$, then $D_{1}=\overleftrightarrow{K}_{1}$

(b) if $n_{1}=2$, then $D_{1}$ is any of digraphs depicted on Figure 5,

(c) if $n_{1}=3$, then $D_{1}$ is 1-regular digraph,

(d) if $n_{1} \geq 4$, then there is no digraph $D_{1}$ such that the statement given by the proposition holds.

Proof. Let us denote by $V\left(D_{1}\right)=\left\{u_{1}, u_{2}, \ldots, u_{n_{1}}\right\}$ and $V\left(D_{2}\right)=\left\{v_{1}, v_{2}, \ldots, v_{n_{2}}\right\}$ the sets of vertices of digraphs $D_{1}$ and $D_{2}$, respectively.

If $N_{\text {out }}(D)=\left[n_{i j}\right]$ is the signless Laplacian matrix of some multigraph $M$, then by observing its rows $n_{1}+1, n_{1}+2, \ldots, n_{1}+n_{2}$, one can conclude that the number:

$$
\left(1-n_{1}\right) \operatorname{outdeg}_{D_{2}}\left(v_{p}\right)-\sum_{q=1, q \neq p}^{n_{2}} n_{p q}\left(N_{\text {out }}\left(D_{2}\right)\right)
$$




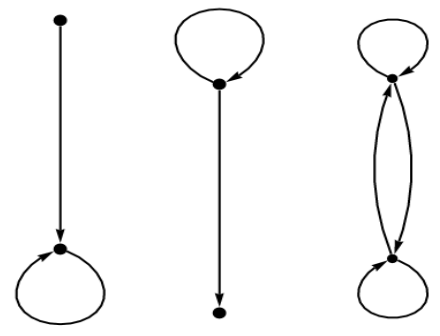

Figure 5: Digraphs from Proposition 5.15

for each $p=1,2, \ldots, n_{2}$, is zero or even positive integer. This means that $n_{1}=1$ and $D_{2}$ is a digraph such that there are no vertices with the common out-neighbours or $D_{2}$ is an isolated vertex.

In the former case, by observing rows $1,2, \ldots, n_{1}$ of $N_{\text {out }}(D)$, one concludes that:

$$
\operatorname{outdeg}_{D_{1}}\left(u_{k}\right)+n_{2}-m_{2},
$$

for each $k=1,2, \ldots, n_{1}$, is zero or even positive integer. Here $m_{2}$ is the number of edges of $D_{2}$, and the proof for statements 1 . and 2. follows.

If $D_{2}$ is an isolated vertex, then by observing rows $1,2, \ldots, n_{1}$ of $N_{\text {out }}(D)$, we get that:

$$
\operatorname{outdeg}_{D_{1}}\left(u_{k}\right)-\sum_{l=1, l \neq k}^{n_{1}} n_{k l}\left(N_{\text {out }}\left(D_{1}\right)\right)-n_{1}+2,
$$

for each $k=1,2, \ldots, n_{1}$, is zero or even positive integer. Let us consider the structure of $D_{1}$.

If $n_{1}=1$ or $n_{1}=2$, statements $(a)$ and $(b)$ follows from (5.1) by direct computation.

If $n_{1}=3$, then $3 \geq$ outdeg $_{D_{1}}\left(u_{k}\right) \geq 1$ must hold for each $k=1,2,3$. Let us suppose that outdeg $_{D_{1}}\left(u_{1}\right)=3$. This implies $\operatorname{indeg}_{D_{1}}\left(u_{1}\right)=\operatorname{indeg}_{D_{1}}\left(u_{2}\right)=\operatorname{indeg}_{D_{1}}\left(u_{3}\right)=1$, and since the out-degree of $u_{2}$ and $u_{3}$ must be at least $1,(5.1)$ will be a negative number for at least one $k$. One can analyse the case when outdeg $_{D_{1}}\left(u_{1}\right)=2$ the same way. And finally, if outdeg $g_{D_{1}}\left(u_{1}\right)=1,(5.1)$ is a non-negative integer if and only if $\sum_{l=2}^{3} n_{1 l}\left(N_{\text {out }}\left(D_{1}\right)\right)=0$. Since the out-degree of each vertex in $D_{1}$ must be at least 1 , $D_{1}$ is 1-regular digraph.

Now, we will prove that there is no digraph $D_{n_{1}}$ of order $n_{1} \geq 4$ such that (5.1) is zero or even positive integer. The proof will be carried out by use of the mathematical induction on the number of vertices $n_{1}$ of $D_{n_{1}}$.

If $n_{1}=4$, analogously as in the case when $n_{1}=3$, one can show that there is at least one vertex, for example $u_{k}$, in $D_{4}$ such that outdeg $_{D_{4}}\left(u_{k}\right)<\sum_{l=1, l \neq k}^{4} n_{k l}\left(N_{\text {out }}\left(D_{4}\right)\right)+2$, where $k \in\{1,2,3,4\}$. Let us suppose that in a digraph $D_{s}$ of order $s>4$ there is at least one vertex such that (5.1) is a negative number. Let us consider a digraph $D_{s+1}$ of order $s+1$. By deleting an arbitrary vertex of $D_{s+1}$ we get a digraph $D_{s}$ of order $s$, where, according to the inductive hypothesis, we can find at least one vertex, say $u_{x}$, such that

$$
\operatorname{outdeg}_{D_{s}}\left(u_{x}\right)<\sum_{q=1, q \neq p}^{s} n_{x q}\left(N_{\text {out }}\left(D_{s}\right)\right)+s-2 \text {. }
$$


If we return the removed vertex and all edges that are incident to it, we get the following inequalities:

$$
\begin{gathered}
\text { outdeg }_{D_{s+1}}\left(u_{x}\right) \leq \operatorname{outdeg}_{D_{s}}\left(u_{x}\right)+1< \\
\sum_{q=1, q \neq i}^{s} n_{x q}\left(N_{\text {out }}\left(D_{s}\right)\right)+s-2+1 \leq \sum_{q=1, q \neq p}^{s+1} n_{x q}\left(N_{\text {out }}\left(D_{s+1}\right)\right)+s-1 .
\end{gathered}
$$

Hence, according to the principle of the mathematical induction, when $D_{2}$ is an isolated vertex, there is no digraph $D_{1}$ of order $n_{1} \geq 4$ such that $N_{\text {out }}(D)=N_{\text {out }}\left(D_{1} \nabla_{\text {out }} D_{2}\right)=$ $Q(M)$.

Proposition 5.16. Let $D=D_{1} \nabla D_{2}$ be the digraph obtained by joining two connected disjoint digraphs $D_{1}$ and $D_{2}$ of orders $n_{1}$ and $n_{2}$, respectively. If $N_{\text {out }}(D)$ is the signless Laplacian matrix of some multigraph, then:

1. $D_{1}$ is an isolated vertex, while $D_{2}$ is any of digraphs depicted on Figure 6;

2. $D_{1}=D_{2}=\overleftrightarrow{K}_{1}$;

3. there are no digraphs $D_{1}$ and $D_{2}$ of orders $n_{1}, n_{2} \geq 3$ such that the statement given by the proposition holds.
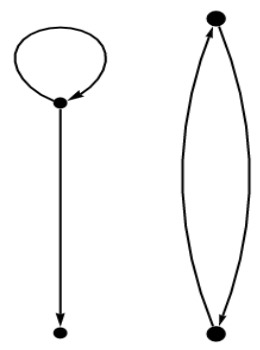

Figure 6: Digraphs from Proposition 5.16

Proof. Let us denote by $V\left(D_{1}\right)=\left\{v_{1}, v_{2}, \ldots, v_{n_{1}}\right\}$ and $V\left(D_{2}\right)=\left\{u_{1}, u_{2}, \ldots, u_{n_{2}}\right\}$ the sets of vertices of digraphs $D_{1}$ and $D_{2}$, respectively. We have:

$$
\begin{gathered}
N_{\text {out }}(D)=N_{\text {out }}\left(D_{1} \nabla D_{2}\right)=\left(\begin{array}{cc}
A_{1} & J^{T} \\
J & A_{2}
\end{array}\right)\left(\begin{array}{cc}
A_{1}^{T} & J^{T} \\
J & A_{2}^{T}
\end{array}\right)= \\
\left(\begin{array}{cc}
N_{\text {out }}\left(D_{1}\right)+J^{T} J & A_{1} J^{T}+J^{T} A_{2}^{T} \\
\left(A_{1} J^{T}+J^{T} A_{2}^{T}\right)^{T} & N_{\text {out }}\left(D_{2}\right)+J J^{T}
\end{array}\right),
\end{gathered}
$$

where $A_{1}$ and $A_{2}$ are the adjacency matrices of digraphs $D_{1}$ and $D_{2}$, respectively.

If $N_{\text {out }}(D)=\left[n_{i j}\right]$ is the signless Laplacian matrix of some multigraph, we have:

$$
\left(1-n_{2}\right) \operatorname{outdeg}_{D_{1}}\left(v_{i}\right)+\left(2-n_{1}\right) n_{2}-\sum_{j=1, j \neq i}^{n_{1}} n_{i j}\left(N_{\text {out }}\left(D_{1}\right)\right)-m_{2}=2 w_{1},
$$

for some non-negative integer $w_{1}$ and $i=1,2, \ldots, n_{1}$, and

$$
\left(1-n_{1}\right) \text { outdeg }_{D_{2}}\left(u_{k}\right)+n_{1}\left(2-n_{2}\right)-\sum_{l=1, l \neq k}^{n_{2}} n_{i j}\left(N_{\text {out }}\left(D_{2}\right)\right)-m_{1}=2 w_{2},
$$


for some non-negative integer $w_{2}$ and $k=1,2, \ldots, n_{2}$, where $m_{1}$ and $m_{2}$ are the numbers of edges of digraphs $D_{1}$ and $D_{2}$, respectively.

First, let us prove that $n_{1}<3$. Since (5.2) means that:

$$
\left(1-n_{2}\right) \operatorname{outdeg}_{D_{1}}\left(v_{i}\right) \geq\left(n_{1}-2\right) n_{2}+\sum_{j=1, j \neq i}^{n_{1}} n_{i j}\left(N_{\text {out }}\left(D_{1}\right)\right)+m_{2}
$$

holds for each $i=1,2, \ldots, n_{1}$, if we suppose that $n_{1} \geq 3$, we get:

$$
0 \geq 1+\sum_{j=1, j \neq i}^{n_{1}} n_{i j}\left(N_{\text {out }}\left(D_{1}\right)\right)+m_{2}
$$

that is a contradiction. In the same way, one can prove that $n_{2}<3$.

Statements 1 . and 2. from the proposition one can get by direct analysis of (5.2) and (5.3).

\section{References}

[1] J. A. Anderson, Discrete mathematics with combinatorics, Prentice Hall, 2nd edition, 2004.

[2] J. Bang-Jensen and G. Z. Gutin, Digraphs. Theory, algorithms and applications, London: Springer, 1st edition, 2000.

[3] M. Behzad, G. Chartrand and L. Lesniak-Foster, Graphs and digraphs, Wadsworth International Group, 1st edition, 1981.

[4] A. Berman and R. J. Plemmons, Nonnegative matrices in the mathematical sciences, Academic Press, 1979.

[5] R. A. Brualdi, Spectra of digraphs, Linear Algebra Appl. 432 (2010), 2181-2213, doi:10.1016/ j.laa.2009.02.033, http://dx.doi.org/10.1016/j.laa.2009.02.033.

[6] D. Cvetković, P. Rowlinson and S. Simić, An introduction to the theory of graph spectra, volume 75 of London Mathematical Society Student Texts, Cambridge University Press, Cambridge, 2010.

[7] D. Cvetković and S. K. Simić, Towards a spectral theory of graphs based on the signless Laplacian. II, Linear Algebra Appl. 432 (2010), 2257-2272, doi:10.1016/j.laa.2009.05.020, http://dx.doi.org/10.1016/j.laa.2009.05.020.

[8] D. M. Cvetković, M. Doob and H. Sachs, Spectra of graphs - Theory and applications, Johann Ambrosius Barth, Heidelberg, 3rd edition, 1995.

[9] B. Elspas and J. Turner, Graphs with circulant adjacency matrices, J. Combinatorial Theory 9 (1970), 297-307.

[10] C. Godsil and G. Royle, Algebraic graph theory, volume 207 of Graduate Texts in Mathematics, Springer-Verlag, New York, 2001, doi:10.1007/978-1-4613-0163-9, http://dx. doi . org/10.1007/978-1-4613-0163-9.

[11] J. M. Kleinberg, Authoritative sources in a hyperlinked environment, in: Proceedings of the Ninth Annual ACM-SIAM Symposium on Discrete Algorithms (San Francisco, CA, 1998), ACM, New York, 1998 pp. 668-677.

[12] A. N. Langville and C. D. Meyer, A survey of eigenvector methods for Web information retrieval, SIAM Rev. 47 (2005), 135-161, doi:10.1137/S0036144503424786, http://dx. doi.org/10.1137/s0036144503424786. 
[13] H. S. Ramane, H. B. Walikar, S. B. Rao, B. D. Acharya, P. R. Hampiholi, S. R. Jog and I. Gutman, Spectra and energies of iterated line graphs of regular graphs, Appl. Math. Lett. 18 (2005), 679-682, doi:10.1016/j.aml.2004.04.012, http://dx.doi.org/10.1016/ j.aml.2004.04.012.

[14] D. Randall, A. O'Neill and A. Irani, CS 6550 - Design and analysis of algorithms, Lecture and notes, 2005, http://people.math.gatech.edu/ randall/AlgsE05/ nov14.pdf.

[15] D. A. Spielman, Spectral graph theory and its applications, in: FOCS '07 Proceedings of the 48th Annual IEEE Symposium on Foundations of Computer Science, 2007 pp. 29-38. 